\title{
A NEW SCALING RELATION FOR H II REGIONS IN SPIRAL GALAXIES: UNVEILING THE TRUE NATURE OF THE MASS-METALLICITY RELATION*
}

\author{
F. F. Rosales-Ortega ${ }^{1,3}$, S. F. Sánchez ${ }^{2,3}$, J. Iglesias-Páramo ${ }^{2,3}$, A. I. Díaz ${ }^{1}$, J. M. Vílchez ${ }^{2}$, \\ J. Bland-Hawthorn ${ }^{4}$, B. HusemanN ${ }^{5}$, AND D. Mast ${ }^{2,3}$ \\ ${ }^{1}$ Departamento de Física Teórica, Universidad Autónoma de Madrid, E-28049 Madrid, Spain; frosales@cantab.net \\ 2 Instituto de Astrofísica de Andalucía (CSIC), Camino Bajo de Huétor s/n, Aptdo. 3004, E-18080 Granada, Spain \\ ${ }^{3}$ Centro Astronómico Hispano Alemán, Calar Alto, CSIC-MPG, C/Jesús Durbán Remón 2-2, E-04004 Almeria, Spain \\ ${ }^{4}$ Sydney Institute for Astronomy, School of Physics A28, University of Sydney, NSW 2006, Australia \\ ${ }^{5}$ Leibniz-Institut für Astrophysik Potsdam (AIP), An der Sternwarte 16, D-14482 Potsdam, Germany \\ Received 2012 May 21; accepted 2012 July 25; published 2012 August 22
}

\begin{abstract}
We demonstrate the existence of a local mass, metallicity, star formation relation using spatially resolved optical spectroscopy of $\mathrm{H}$ II regions in the local universe. One of the projections of this distribution-the local mass-metallicity relation-extends over a wide range in this parameter space: three orders of magnitude in mass and a factor of eight in metallicity. We explain the new relation as the combined effect of the differential distributions of mass and metallicity in the disks of galaxies, and a selective star formation efficiency. We use this local relation to reproduce - with a noticeable agreement - the mass-metallicity relation seen in galaxies, and conclude that the latter is a scale-up integrated effect of a local relation, supporting the inside-out growth and downsizing scenarios of galaxy evolution.
\end{abstract}

Key words: galaxies: abundances - galaxies: fundamental parameters - galaxies: ISM - galaxies: stellar content techniques: imaging spectroscopy

Online-only material: color figures

\section{INTRODUCTION}

The existence of a strong correlation between stellar mass and gas-phase metallicity in galaxies is a well-known fact (Lequeux et al. 1979). These parameters are two of the most fundamental physical properties of galaxies, both directly related to the process of galaxy evolution. The mass-metallicity $(\mathcal{M}-Z)$ relation is consistent with more massive galaxies being more metal-enriched. It was established observationally by Tremonti et al. (2004, hereafter T04), who found a tight correlation spanning over 3 orders of magnitude in mass and a factor of 10 in metallicity, using a large sample of star-forming galaxies up to $z \sim 0.1$ from the Sloan Digital Sky Survey (SDSS). The $\mathcal{M}-Z$ relation appears to be independent of large-scale environment (Mouhcine et al. 2007) and has been established at all accessible redshifts (e.g., Savaglio et al. 2005; Erb et al. 2006; Maiolino et al. 2008).

Considerable work has been devoted to understanding the physical mechanisms underlying the $\mathcal{M}-Z$ relation. The proposed scenarios to explain its origin can be broadly categorized as: (1) the loss of enriched gas by outflows (T04; Kobayashi et al. 2007); (2) the accretion of pristine gas by inflows (Finlator \& Davé 2008); (3) variations of the initial mass function with mass (Köppen et al. 2007); (4) selective star formation efficiency or downsizing (Brooks et al. 2007; Ellison et al. 2008; Calura et al. 2009; Asari et al. 2009); or a combination of these. Recent studies also show evidence of a relation with star formation rate (SFR) inferring a fundamental $\mathcal{M}-Z-$ SFR relation (hereafter FMR; Lara-López et al. 2010; Mannucci et al. 2010; Yates et al. 2012).

As yet, there has been no major effort to test the $\mathcal{M}-Z$ relation using spatially resolved information. The most likely

\footnotetext{
* Based on observations collected at the Centro Astronómico

Hispano-Alemán (CAHA) at Calar Alto, operated jointly by the Max-Planck Institut für Astronomie and the Instituto de Astrofísica de Andalucía (CSIC).
}

example might be ascribed to Edmunds \& Pagel (1984) and Vila-Costas \& Edmunds (1992) who noticed a correlation between surface mass density and gas metallicity in a number of galaxies. Nowadays, the imaging spectroscopy technique can potentially prove to be the key to understanding many of the systematic effects that hamper the role of the distribution of mass and metals in galaxies. In this work, we use integral field spectroscopy (IFS) observations of a sample of nearby galaxies to demonstrate (1) the existence of a local $\mathcal{M}-Z$-star-formation relation and (2) how the global $\mathcal{M}-Z$ relation seen in galaxies can be reproduced by the presence of the local one. We present convincing evidence that the observed $\mathcal{M}-Z$ relations represent a simple sequence in astration.

\section{DATA SAMPLE AND ANALYSIS}

The study was performed using IFS data of a sample of nearby disk galaxies, part belonging to the PINGS survey (RosalesOrtega et al. 2010), and a sample of face-on spiral galaxies from Mármol-Queraltó et al. (2011) as part of the feasibility studies for the CALIFA survey (Sánchez et al. 2012a). The observations were designed to obtain continuous coverage spectra of the whole surface of the galaxies. The final sample is comprised of 38 objects, with a redshift range between $\sim 0.001-0.025$. Although this sample is by no means a statistical subset of the galaxies in the local universe, it is a representative sample of face-on, mostly quiescent, spiral galaxies at the considered redshift range. They were observed with the PMAS spectrograph (Roth et al. 2005) in the PPAK mode (Verheijen et al. 2004; Kelz et al. 2006) on the $3.5 \mathrm{~m}$ telescope in Calar Alto with similar setup, resolutions, and integration times, covering their optical extension up to $\sim 2.4$ effective radii within a wavelength range $\sim 3700-7000 \AA$. Data reduction was performed using R3D (Sánchez 2006), obtaining as an output a data cube for each galaxy, with a final spatial sampling between 1-2 $\operatorname{arcsec}$ pixel $^{-1}$, 
which translates to a linear physical size between a few hundreds of parsecs to $\sim 1 \mathrm{kpc}$ (depending on the size of the object). Details on the sample, observing strategy, setups, and data reduction can be found in Rosales-Ortega et al. (2010) and Mármol-Queraltó et al. (2011).

The $\mathrm{H}$ II regions in these galaxies were detected, spatially segregated, and spectrally extracted using HIIEXPLORER (Sánchez et al. 2012b). We detected a total of $2573 \mathrm{H} \mathrm{II} \mathrm{regions} \mathrm{with} \mathrm{good}$ spectroscopic quality. This is by far the largest spatially resolved, nearby spectroscopic HiI region survey ever accomplished. Note that for the more distant galaxies the segregated $\mathrm{H}$ in regions may be comprised of a few classical ones, i.e., H II aggregations, which may not be useful in analyzing additive/integrated properties as in individual $\mathrm{H}$ II regions (e.g., H $\alpha$ luminosity function), but are perfectly suited for the study of line ratios and chemical abundances. The emission lines were decoupled from the underlying stellar population using FIT3D (Sánchez et al. 2007), following a robust and well-tested methodology (Rosales-Ortega et al. 2010; Sánchez et al. 2011). Extinction-corrected flux intensities of the stronger emission lines were obtained and used to select only star-forming regions based on typical BPT diagnostic diagrams (Baldwin et al. 1981) using a combination of the Kewley et al. (2001) and Kauffmann et al. (2003) demarcation curves. Our final sample is comprised of 1896 high-quality, spatially resolved $\mathrm{H}$ II regions/aggregations of disk galaxies in the local universe. Details on the procedure can be found in Sánchez et al. (2012b).

Gas-phase oxygen abundances were estimated using the O3N2 calibrator (Pettini \& Pagel 2004), based on the [O III], $\mathrm{H} \beta$, [N II], and $\mathrm{H} \alpha$ emission lines. We use this indicator because it is less dependent on dust attenuation and we could use the whole HII region sample (given the lack of [O II] $\lambda 3727$ in some galaxies due to redshift). We used the prescriptions given by Bell $\&$ de Jong (2001) to convert $B-V$ colors into a $B$-band mass-to-light ratio $(M / L)$ to derive the (luminosity) surface mass density $\left(\Sigma_{\mathrm{Lum}}, M_{\odot} \mathrm{pc}^{-2}\right)$ within the area encompassed by our IFS-segmented $\mathrm{H}$ II regions. The $B$ - and $V$-band surface brightness within the considered areas were derived directly from the (emission-line-free) IFS data. The estimated error in the derived $\Sigma_{\text {Lum }}$ is $\sim 0.25$ dex, taking into account the error in the flux calibration of our IFS data set (Rosales-Ortega et al. 2010; Mármol-Queraltó et al. 2011), and the expected uncertainty of the Bell \& de Jong (2001) formulation. The relation between the mass surface density and radius was checked by normalizing the mass profile by the effective radius, finding a similar slope for all galaxies closer to unity, i.e., the mass profile follows an exponential law, which is what is expected for disk galaxies (e.g., Bakos et al. 2008). Furthermore, we compared our radial mass profiles with the more sophisticated $K$-band derived profiles of those common galaxies in our sample with the DiskMass Survey (Bershady et al. 2010; Martinsson 2011) finding an agreement within $20 \%$, strengthening the validity of our derived masses.

\section{THE LOCAL $\mathcal{M}-Z$ RELATION}

The left panel of Figure 1 shows the striking correlation between the local surface mass density and gas metallicity for our sample of nearby $\mathrm{H}$ II regions, i.e., the local $\mathcal{M}-Z$ relation, extending over $\sim 3$ orders of magnitude in $\Sigma_{\text {Lum }}$ and a factor $\sim 8$ in metallicity. As in the case of the global $\mathcal{M}-Z$ relation (e.g., T04), the correlation is nearly linear for lower $\Sigma_{\text {Lum }}$, flattening gradually at higher values. Also remarkable is the tightness of the correlation; the $1 \sigma$ scatter of the data about the median is \pm 0.14 dex. The notable similarity with the global $\mathcal{M}-Z$
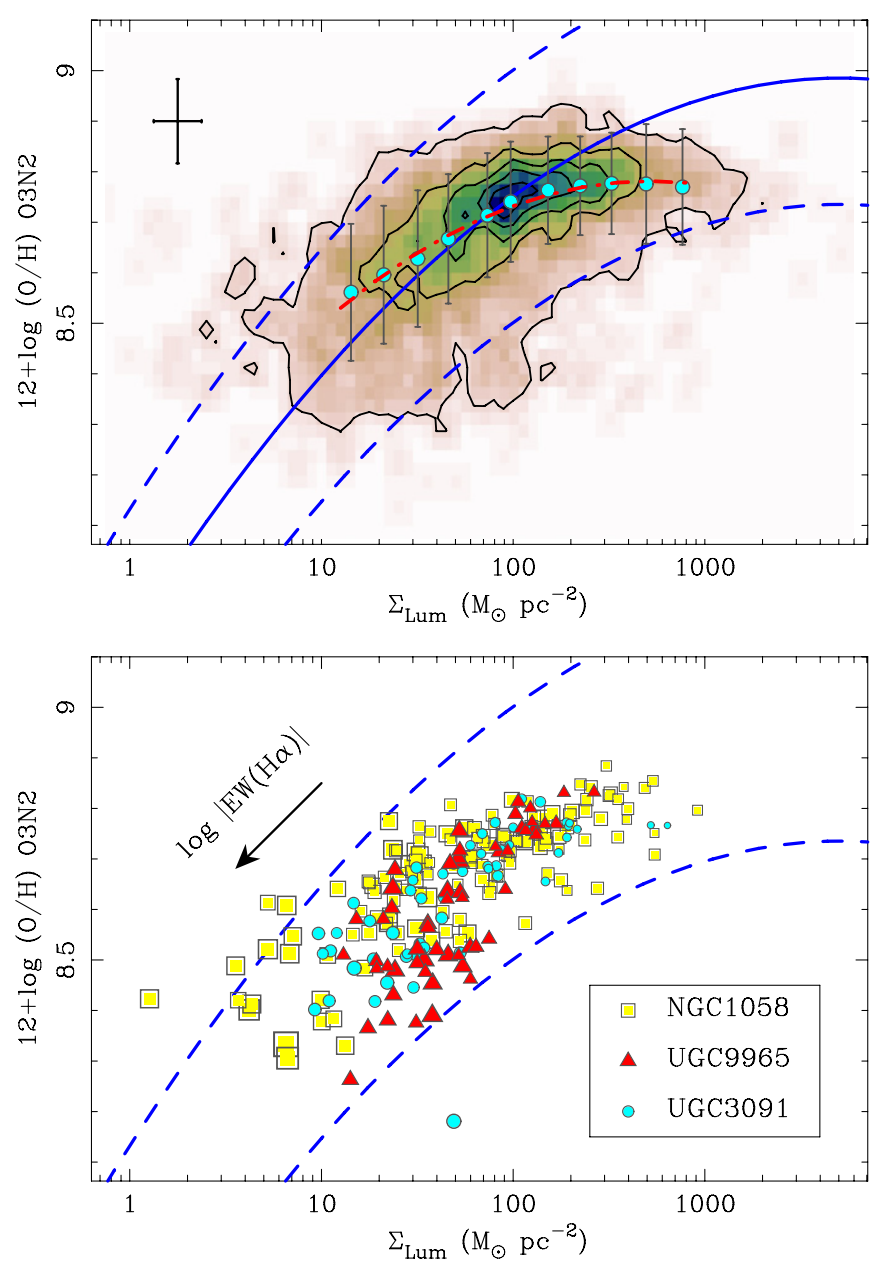

Figure 1. Left panel: the relation between surface mass density and gas-phase oxygen metallicity for $\sim 2000 \mathrm{H}$ II regions in nearby galaxies, the local $\mathcal{M}-Z$ relation. The first contour stands for the mean density value, with a regular spacing of four times this value for each consecutive contour. The blue circles represent the mean (plus $1 \sigma$ error bars) in bins of 0.15 dex. The red dasheddotted line is a polynomial fit to the data. The blue lines correspond to the T04 relation $( \pm 0.2$ dex $)$ scaled to the relevant units. Typical errors for $\Sigma_{\text {Lum }}$ and metallicity are represented. Right panel: distribution of $\mathrm{H}$ II regions along the local $\mathcal{M}-Z$ relation for three galaxies of the sample at different redshifts. The size of the symbols are linked to the value of $|\mathrm{EW}(\mathrm{H} \alpha)|$, being inversely proportional to $\Sigma_{\text {Lum }}$ and metallicity as shown.

(A color version of this figure is available in the online journal.)

relation can be visually recognized with the aid of the blue lines which stand for the T04 fit $( \pm 0.2 \mathrm{dex})$ to the global $\mathcal{M}-Z$ relation, shifted arbitrarily both in mass $(\sim 5 \mathrm{mag}$; to account for the difference in size between galaxies and $\mathrm{H}$ II regions) and metallicity $(-0.15 \mathrm{dex}$; due to the well-known effect of metallicity scale) to coincide with the peak of the H II region $\mathcal{M}-Z$ distribution, which clearly follows the T04 relationship. With this offset, the local $\mathcal{M}-Z$ values stand within $90 \%$ of the $95 \%$ range of the T04 relation. A polynomial fit of the $\Sigma_{\text {Lum }}$ versus metallicity relation yields

$$
\begin{aligned}
12+\log (\mathrm{O} / \mathrm{H})_{\mathrm{O} 3 \mathrm{~N} 2}= & 8.079_{ \pm 0.141}+0.525_{ \pm 0.143} \Sigma_{\mathrm{Lum}} \\
& -0.098_{ \pm 0.035}\left[\Sigma_{\text {Lum }}\right]^{2}
\end{aligned}
$$

valid over the range $1<\log \Sigma_{\text {Lum }}<3$. We obtain the same shape of the relation (and similar fit) if we adopt the $R_{23}$ metallicity calibration of T04, but with a higher scatter $(\sim 20 \%)$ due to the lowest number of $\mathrm{H}$ II regions in our sample with the $\left[\mathrm{O}_{\mathrm{II}}\right] \lambda 3727$ line. Other typical calibrations were tested finding 


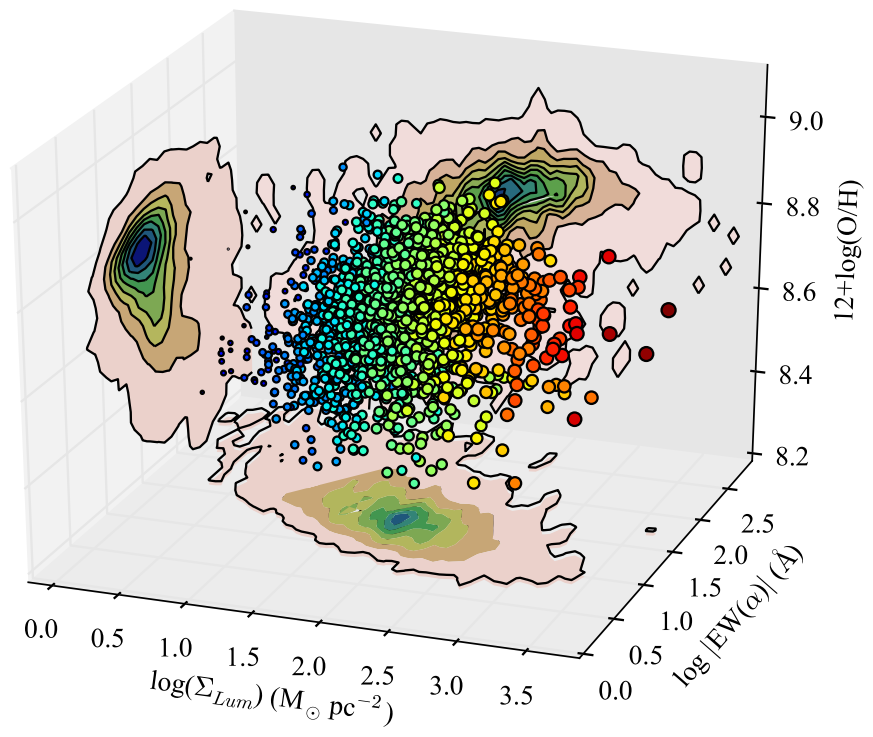

Figure 2. 3D representation of the local $\mathcal{M}-Z-\mathrm{EW}(\mathrm{H} \alpha)$ relation. The size and color scaling of the data points are linked to the value of $\log \Sigma_{\text {Lum }}$ (i.e., low-blue to high-red values). The projection of the data over any pair of axes reduces to the local $\mathcal{M}-Z, \mathcal{M}-\mathrm{EW}(\mathrm{H} \alpha)$, and metallicity- $\mathrm{EW}(\mathrm{H} \alpha)$ relations. An online 3D animated version is available at http://tinyurl.com/local-MZ-relation.

(A color version of this figure is available in the online journal.)

similar results (e.g., N2; Denicoló et al. 2002), i.e., the scale of the relation changes when using different indicators, but the overall, qualitative shape does not change.

Note that the local $\mathcal{M}-Z$ relation holds for individual galaxies with a large-enough dynamical range in $\Sigma_{\text {Lum }}$ and metallicity to cover the whole parameter space of the relation, i.e., it is indeed a local relationship. This is shown in the right panel of Figure 1, displaying the local $\mathcal{M}-Z$ relation for the $\mathrm{H}$ II regions of three galaxies in our sample at different redshifts. For the closest galaxy (NGC 1058; $z \sim 0.0017$ ), the relationship holds for $\sim 3$ mag in $\Sigma_{\text {Lum }}$, and even with the loss of spatial resolution at higher redshifts the relation persists over a large dynamic range.

In addition, we find the existence of a more general relation between mass surface density, metallicity, and the equivalent width of $\mathrm{H} \alpha$, defined as the emission-line luminosity normalized to the adjacent continuum flux, i.e., a measure of the SFR per unit luminosity (Kennicutt 1998). This functional relation is evident in a three-dimensional (3D) space with orthogonal coordinate axes defined by these parameters, consistent with $|\mathrm{EW}(\mathrm{H} \alpha)|$ being inversely proportional to both $\Sigma_{\text {Lum }}$ and metallicity, as shown in Figure 2. The projection of the local $\mathcal{M}-Z-\mathrm{EW}(\mathrm{H} \alpha)$ relation on the planes defined in this $3 \mathrm{D}$ space correspond to the local $\mathcal{M}-Z, \mathcal{M}-\mathrm{EW}(\mathrm{H} \alpha)$, and metallicity- $\mathrm{EW}(\mathrm{H} \alpha)$ relations. Figure 3 shows the projection of $\log |\mathrm{EW}(\mathrm{H} \alpha)|$ as a strong and tight function of $\Sigma_{\text {Lum }}$ extending over more than three orders of magnitude. The projection on the $|\mathrm{EW}(\mathrm{H} \alpha)|$ versus metallicity plane shows an existent but weaker correlation.

\section{THE GLOBAL $\mathcal{M}-Z$ RELATION}

In order to test whether the global $\mathcal{M}-Z$ relation observed by T04 using SDSS data is a reflection (aperture effect) of the local $\mathrm{H}$ II region mass density versus metallicity relation, we perform the following exercise. We simulate a galaxy with typical $M_{B}$ and $B-V$ values drawn from flat distributions in magnitude $(-15$ to -23$)$ and color $(\sim 0.4-1)$. A redshift is assumed for the mock galaxy, drawn from a Gaussian distribution with mean

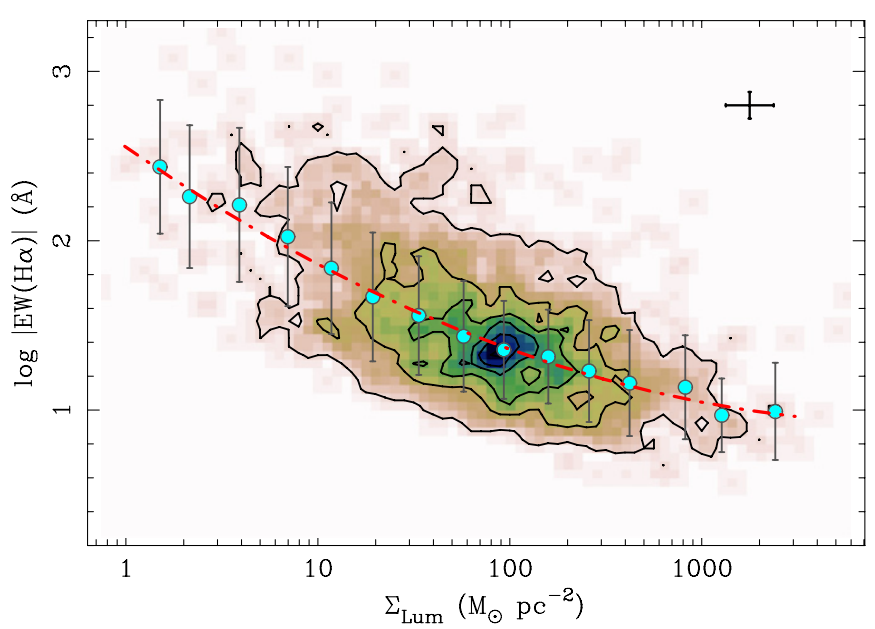

Figure 3. Relation between $|\mathrm{EW}(\mathrm{H} \alpha)|$ and surface mass density, i.e., a projection of the local $\mathcal{M}-Z-\mathrm{EW}(\mathrm{H} \alpha)$ relation. Contours and symbols as in Figure 1. The red line is a polynomial fit to the data.

(A color version of this figure is available in the online journal.)

$\sim 0.1$ and $\sigma=0.05$, with a redshift cut $0.02<z<0.3$ in order to resemble the SDSS T04 distribution. The mass of the galaxy is derived using the integrated $B$-band magnitudes, $B-V$ colors and the average $M / L$ ratio following Bell \& de Jong (2001). The effective radius of the mock galaxy is estimated using the well-known luminosity-scale relation (e.g., Brooks et al. 2011) assuming a normal standard deviation of $0.3 \mathrm{dex}$ (Shen et al. 2003). Once the mass and effective radius are known, the surface brightness at the center of the mock galaxy is derived assuming an exponential light distribution. Then, the metallicity is calculated at different radii up to an aperture equal to the SDSS fiber ( 3 arcsec) in 100 bins using Equation (1), i.e., the metallicity that corresponds to the mass density surface at each bin. The metallicity assigned to the mock galaxy is the mean value determined within the aperture, assuming an error equal to the standard deviation of the derived distribution plus a systematic error of $\sim 0.1 \mathrm{dex}$, intrinsic to the derivation of the metallicity. We used the O3N2 metallicity conversion by Kewley \& Ellison (2008, hereafter K08) to convert to the T04 base. The process is repeated over 10,000 times in order to obtain a reliable distribution in the mass and metallicity of the mock galaxies.

Figure 4 shows the result of the simulation, i.e., the distribution of the mock galaxies in the $\mathcal{M}-Z$ parameter space. We reproduce the overall shape of the global $\mathcal{M}-Z$ relation assuming a local $\mathcal{M}-Z$ relation and considering the aperture effect of the SDSS fiber, over $\sim 5$ orders of magnitude in mass and $\sim 1.5$ in metallicity. The correspondence can be clearly seen by comparing the distribution of points with the overlaid lines corresponding to the T04 fit (black) and the K08 \pm 0.2 dex relation (blue), for which the agreement is extremely good over a wide range of masses. The deviations of the T04 relation at the high-mass end are somewhat expected given that the choice of this metallicity calibrator has a significant effect on the $y$-intercept of the $\mathcal{M}-Z$ relation (K08). The result is remarkable considering that we are able to reproduce the global $\mathcal{M}-Z$ relation over a huge dynamical range, using a local $\mathcal{M}-Z$ relation derived from a galaxy sample with a restricted range in mass $\left(9.2<\log M_{\text {Lum }}<11.2\right)$ and metallicity $(8.3<12+$ $\log (\mathrm{O} / \mathrm{H})<8.9)$, indicated by the rectangle shown in Figure 4. 


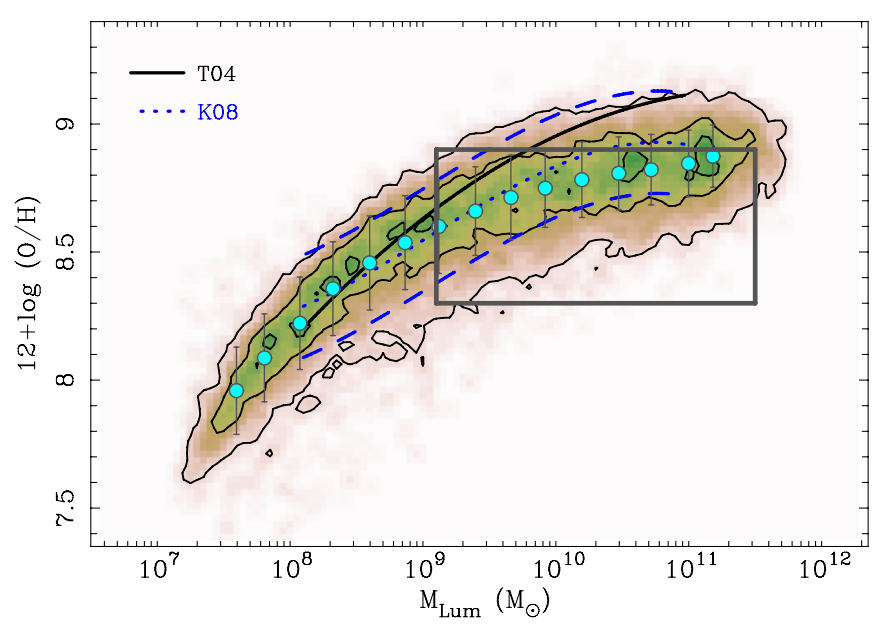

Figure 4. Distribution of simulated galaxies in the $\mathcal{M}-Z$ plane assuming a local $\mathcal{M}-Z$ relation and considering the aperture effect of the SDSS fiber, as explained in the text. The contours correspond to the density of points, while the circles represent the mean value (plus $1 \sigma$ error bars) in bins of 0.15 dex. The black line stands for the T04 fitting, while the blue lines correspond to the K08 \pm 0.2 dex relation. The rectangle encompasses the range in mass and metallicity of the galaxy sample of this work.

(A color version of this figure is available in the online journal.)

\section{ON THE ORIGIN OF THE LOCAL AND GLOBAL $\mathcal{M}-Z$ RELATIONS}

We interpret the local $\mathcal{M}-Z-\mathrm{EW}(\mathrm{H} \alpha)$ relation as the consequence of a more subtle relation between the mass, metallicity, and star formation history with galaxy radius. Galaxies are known to have radial gradients in their physical properties (e.g., Searle 1971; McCall et al. 1985; Vila-Costas \& Edmunds 1992; Zaritsky et al. 1994; Bell \& de Jong 2000). The fact that gas metallicity increases with mass surface density is just reflecting the existence of radial metallicity gradients and radial surface mass density gradients in spirals. On the other hand, the $|\mathrm{EW}(\mathrm{H} \alpha)|$ is a parameter that scales with the SFR per unit mass, i.e., the specific SFR (SSFR) and is a proxy of the stellar birthrate parameter $b$-Scalo, which is the ratio of the present to past-average SFR (Kennicutt et al. 1994; Kennicutt 1998). Multiphase chemical evolution models for spiral disks show that both the distributions of neutral and molecular gas show maxima that move from the center outward through the disk as the galaxy evolves. Accordingly, the maximum of the star formation activity, very high in the central regions at early times, also moves throughout the disk as the gas in the central region is very efficiently consumed. This leads to high present-day abundances and low current SSFRs in the center and radial abundance gradients that flatten with time. The location on the disk of the maxima in the gas distributions at the present time, which reflects the degree of evolution, is related to the galaxy morphological type with early-type galaxies showing the peak of the star formation at outer regions compared to late-type galaxies, which implies a higher degree of evolution and a flatter galaxy for early-type galaxies (Molla et al. 1997; Mollá \& Díaz 2005).

The observed trend agrees qualitatively with this scenario: the inverse gradient of $|\mathrm{EW}(\mathrm{H} \alpha)|$ versus $\Sigma_{\text {Lum }}$ or metallicity would reflect an evolutionary sequence across the disk of the galaxies, i.e., lower (inner) values of $|\mathrm{EW}(\mathrm{H} \alpha)|$ corresponding to lower SSFR, and vice versa. In this scenario, the inner regions of the galaxy form first and faster, increasing the gas metallicity of the surrounding interstellar medium. As the galaxy evolves and grows with time, the star formation progresses radially, creating a radial metallicity gradients in the disk of spirals. Mass is progressively accumulated at the inner regions of the galaxy, raising the surface mass density and creating a bulge, with corresponding high metallicity values but low SSFR (low $|\mathrm{EW}(\mathrm{H} \alpha)|)$, i.e., an "inside-out" galaxy disk growth. In such a case, the local $\mathcal{M}-Z$ relation would reflect a more fundamental relation between mass, metallicity, and star formation efficiency as a function of radius, equivalent to a local downsizing effect.

Following this reasoning, the origin of the global $\mathcal{M}-Z$ relation can be explained as the combined effect of the existence of the local $\mathcal{M}-Z$ relation, and (as a second-order effect) an aperture bias due to the different covering factors of the SDSS fiber, as suggested by the exercise performed in Section 4. On the other hand, although we cannot reproduce the FMR in our simulation given that there is no empirical way of determining an SFR of the mock galaxies, the existence of the FMR could also be interpreted as a scaled-up version of the local $\mathcal{M}-Z-S S F R$ relation. Furthermore, under the proposed scenario, the flattening of the $\mathcal{M}-Z$ relation at the high-mass end could be explained as the combined observational effect of: (1) an intrinsic saturation of the enriched gas due to the low SSFR in the inner regions of the galaxies; (2) a deviation from linearity of the abundance gradients in the center of spiral galaxies, i.e., a flattening (or drop) in the metallicity gradient at the innermost radii as suggested by recent works (e.g., RosalesOrtega et al. 2011; Bresolin et al. 2012; Sánchez et al. 2012b) and predicted from chemical evolution models (Molla et al. 1997); and (3) the depletion of the oxygen emission lines in the optical due to high efficiency of cooling in high-metallicity $(12+\log (\mathrm{O} / \mathrm{H})>8.7)$, low-temperature $\left(T_{\mathrm{e}}<10^{4} \mathrm{~K}\right) \mathrm{H}$ II regions.

\section{CONCLUSIONS}

By using the IFS of a sample of nearby galaxies, we demonstrate the existence of a local relation between the surface mass density, gas-phase oxygen abundance, and $|\mathrm{EW}(\mathrm{H} \alpha)|$ in 2000 spatially resolved H II regions of the local universe. The projection of this distribution in the metallicity versus $\Sigma_{\text {Lum }}$ plane-the local $\mathcal{M}-Z$ relation-shows a tight correlation expanding over a wide range in this parameter space. A similar behavior is seen for the $|\mathrm{EW}(\mathrm{H} \alpha)|$ versus $\Sigma_{\text {Lum }}$ relation. In both projections, the value of $|\mathrm{EW}(\mathrm{H} \alpha)|$ is inversely proportional to mass and metallicity. Notably, the local $\mathcal{M}-Z$ relation has the same shape as the global $\mathcal{M}-Z$ relation for galaxies observed by T04. We explain the new relation as the combination of: (1) the well-known relationships between both the mass and metallicity with respect to the differential distributions of these parameters found in typical disk galaxies, i.e., the inside-out growth; and (2) the fact that more massive regions form stars faster (i.e., at higher SFRs), thus earlier in cosmological times, which can be considered a local downsizing effect, similar to the one observed in individual galaxies (e.g., Pérez-González et al. 2008).

We use the local $\mathcal{M}-Z$ relation to reproduce the global $\mathcal{M}-Z$ relation by means of a simple simulation which considers the aperture effects of the SDSS fiber at different redshifts. We conclude that the $\mathcal{M}-Z$ relation in galaxies is a scale-up integrated effect of a local $\mathcal{M}-Z$ relation in the distribution of star-forming regions across the disks of galaxies, i.e., the relationship is not primary, but obtained from the sum of a number of local linear relations (and their deviations) with respect to the galaxy radius. Under these premises, the existence of the FMR in galaxies might be explained by the presence of an intrinsic local $\mathcal{M}-Z-S S F R$ relation, which relates the 
distribution of mass, metallicity, and SFR across the galaxy disks, driven mainly by the history of star formation, within an inside-out growth scenario.

F.F.R.-O. acknowledges the Mexican National Council for Science and Technology (CONACYT) for financial support under the program Estancias Posdoctorales y Sabáticas al Extranjero para la Consolidación de Grupos de Investigación, 2010-2011. A.I.D. thanks the Spanish Plan Nacional de Astronomía program AYA2010-21887 C04-03.

\section{REFERENCES}

Asari, N. V., Stasińska, G., Fernandes, R. C., et al. 2009, MNRAS, 396, L71

Bakos, J., Trujillo, I., \& Pohlen, M. 2008, ApJ, 683, L103

Baldwin, J. A., Phillips, M. M., \& Terlevich, R. 1981, PASP, 93, 5

Bell, E. F., \& de Jong, R. S. 2000, MNRAS, 312, 497

Bell, E. F., \& de Jong, R. S. 2001, ApJ, 550, 212

Bershady, M. A., Verheijen, M. A. W., Swaters, R. A., et al. 2010, ApJ, 716, 198

Bresolin, F., Kennicutt, R. C., \& Ryan-Weber, E. 2012, ApJ, 750, 122

Brooks, A. M., Governato, F., Booth, C. M., et al. 2007, ApJ, 655, L17

Brooks, A. M., Solomon, A. R., Governato, F., et al. 2011, ApJ, 728, 51

Calura, F., Pipino, A., Chiappini, C., et al. 2009, A\&A, 504, 373

Denicoló, G., Terlevich, R., \& Terlevich, E. 2002, MNRAS, 330, 69

Edmunds, M. G., \& Pagel, B. E. J. 1984, MNRAS, 211, 507

Ellison, S. L., Patton, D. R., Simard, L., et al. 2008, ApJ, 672, L107

Erb, D. K., Shapley, A. E., Pettini, M., et al. 2006, ApJ, 644, 813

Finlator, K., \& Davé, R. 2008, MNRAS, 385, 2181

Kauffmann, G., Heckman, T. M., Tremonti, C., et al. 2003, MNRAS, 346, 1055

Kelz, A., Verheijen, M. A. W., Roth, M. M., et al. 2006, PASP, 118, 129

Kennicutt, R. C. 1998, ARA\&A, 36, 189

Kennicutt, R. C., Tamblyn, P., \& Congdon, C. E. 1994, ApJ, 435, 22

Kewley, L. J., Dopita, M. A., Sutherland, R. S., et al. 2001, ApJ, 556, 121

Kewley, L. J., \& Ellison, S. L. 2008, ApJ, 681, 1183
Kobayashi, C., Springel, V., \& White, S. D. M. 2007, MNRAS, 376, 1465

Köppen, J., Weidner, C., \& Kroupa, P. 2007, MNRAS, 375, 673

Lara-López, M. A., Cepa, J., Bongiovanni, A., et al. 2010, A\&A, 521, L53

Lequeux, J., Peimbert, M., Rayo, J. F., et al. 1979, A\&A, 80, 155

Maiolino, R., Nagao, T., Grazian, A., et al. 2008, A\&A, 488, 463

Mannucci, F., Cresci, G., Maiolino, R., et al. 2010, MNRAS, 408, 2115

Mármol-Queraltó, E., Sánchez, S. F., Marino, R. A., et al. 2011, A\&A, 534, 8

Martinsson, T. P. K. 2011, The Distribution of Mass within Spiral Galaxies: Unique Solutions from Gas and Stellar Kinematics, PhD thesis, Univ. Gröningen

McCall, M. L., Rybski, P. M., \& Shields, G. A. 1985, ApJS, 57, 1

Mollá, M., \& Díaz, A. I. 2005, MNRAS, 358, 521

Molla, M., Ferrini, F., \& Diaz, A. I. 1997, ApJ, 475, 519

Mouhcine, M., Baldry, I. K., \& Bamford, S. P. 2007, MNRAS, 382, 801

Pérez-González, P. G., Rieke, G. H., Villar, V., et al. 2008, ApJ, 675, 234

Pettini, M., \& Pagel, B. E. J. 2004, MNRAS, 348, L59

Rosales-Ortega, F. F., Díaz, A. I., Kennicutt, R. C., et al. 2011, MNRAS, 415, 2439

Rosales-Ortega, F. F., Kennicutt, R. C., Sánchez, S. F., et al. 2010, MNRAS, 405, 735

Roth, M. M., Kelz, A., Fechner, T., et al. 2005, PASP, 117, 620

Sánchez, S. F. 2006, Astron. Nachr., 327, 850

Sánchez, S. F., Cardiel, N., Verheijen, M. A. W., et al. 2007, MNRAS, 376,125

Sánchez, S. F., Kennicutt, R. C., de Paz, A. G., et al. 2012a, A\&A, 538, 8

Sánchez, S. F., Rosales-Ortega, F. F., Marino, R. A., et al. 2012b, A\&A, in press (arXiv:1208.1117)

Sánchez, S. F., Rosales-Ortega, F. F., Kennicutt, R. C., et al. 2011, MNRAS, 410,313

Savaglio, S., Glazebrook, K., Borgne, D. L., et al. 2005, ApJ, 635, 260

Searle, L. 1971, ApJ, 168, 327

Shen, S., Mo, H. J., White, S. D. M., et al. 2003, MNRAS, 343, 978

Tremonti, C. A., Heckman, T. M., Kauffmann, G., et al. 2004, ApJ, 613, 898

Verheijen, M. A. W., Bershady, M. A., Andersen, D. R., et al. 2004, Astron. Nachr., 325, 151

Vila-Costas, M. B., \& Edmunds, M. G. 1992, MNRAS, 259, 121

Yates, R. M., Kauffmann, G., \& Guo, Q. 2012, MNRAS, 422, 215

Zaritsky, D., Kennicutt, R. C., \& Huchra, J. P. 1994, ApJ, 420, 87 